\title{
Stability of a Double-Diffusive Interface in the Diffusive Convection Regime
}

\author{
J. R. CARPENTER* \\ Eawag, Swiss Federal Institute of Aquatic Science and Technology, Kastanienbaum, Switzerland \\ T. SOMMER AND A. WÜEST \\ Eawag, Swiss Federal Institute of Aquatic Science and Technology, Kastanienbaum, and Institute \\ of Biogeochemistry and Pollutant Dynamics, Environmental Science, ETH, Zürich, Switzerland
}

(Manuscript received 7 July 2011, in final form 17 November 2011)

\begin{abstract}
In this paper, the authors explore the conditions under which a double-diffusive interface may become unstable. Focus is placed on the case of a cold, freshwater layer above a warm, salty layer [i.e., the diffusive convection (DC) regime]. The "diffusive interface" between these layers will develop gravitationally unstable boundary layers due to the more rapid diffusion of heat (the destabilizing component) relative to salt. Previous studies have assumed that a purely convective-type instability of these boundary layers is what drives convection in this system and that this may be parameterized by a boundary layer Rayleigh number. The authors test this theory by conducting both a linear stability analysis and direct numerical simulations of a diffusive interface. Their linear stability analysis reveals that the transition to instability always occurs as an oscillating diffusive convection mode and at boundary layer Rayleigh numbers much smaller than previously thought. However, these findings are based on making a quasi-steady assumption for the growth of the interfaces by molecular diffusion. When diffusing interfaces are modeled (using direct numerical simulations), the authors observe that the time dependence is significant in determining the instability of the boundary layers and that the breakdown is due to a purely convective-type instability. Their findings therefore demonstrate that the relevant instability in a DC staircase is purely convective.
\end{abstract}

\section{Introduction}

A fundamental result in the study of ocean mixing is that certain configurations of temperature $T$ and salinity $S$ are unstable to small perturbations, despite the fact that the water column is gravitationally stable. This instability is due to the differing rates of diffusion of $T$ and $S$, and has come to be known as double-diffusive (DD) instability. The first stability analysis to demonstrate the basic mechanism of DD was performed by Stern (1960) using linear profiles of $T$ and $S$, bounded above and below (see Fig. 1a, where $T$ and $S$ are plotted in density units). This analysis was subsequently extended by Veronis

\footnotetext{
* Current affiliation: Department of Geology and Geophysics, Yale University, New Haven, Connecticut.
}

Corresponding author address: Jeff Carpenter, Department of Geology and Geophysics, Yale University, 210 Whitney Ave., New Haven, CT 06511.

E-mail: jeffcarp@gmail.com
(1965), Nield (1967), and Baines and Gill (1969), keeping the same linear profiles. These studies found that unstable motions may develop from small perturbations of the $T-S$ profiles, as long as at least one component is in a gravitationally unstable configuration, thus providing the energy source required to drive the instability. Two different cases exist depending on whether $T$ (the faster diffusing component) or $S$ is destabilizing: (i) the salt-fingering case where warm, salty water overlies cool, fresh water or (ii) the diffusive convection (DC) case with cool, fresh water over warm, salty water (Fig. 1).

Conditions favoring DD are found over vast areas of the oceans (You 2002; Kelley et al. 2003), as well as in lakes (Hoare 1966; Schmid et al. 2004; Sánchez and Roget 2007; Boehrer et al. 2009; Schmid et al. 2010), and are often accompanied by a thermohaline staircase structure in the $T$ and $S$ fields. These staircases consist of a sequence of sharp, high-gradient interfaces of $T$ and $S$ surrounded by nearly homogeneous mixed layers. In this study, we shall focus on the stability properties of the "diffusive interface": that is, with cool, fresh water 
(a) Linear profiles

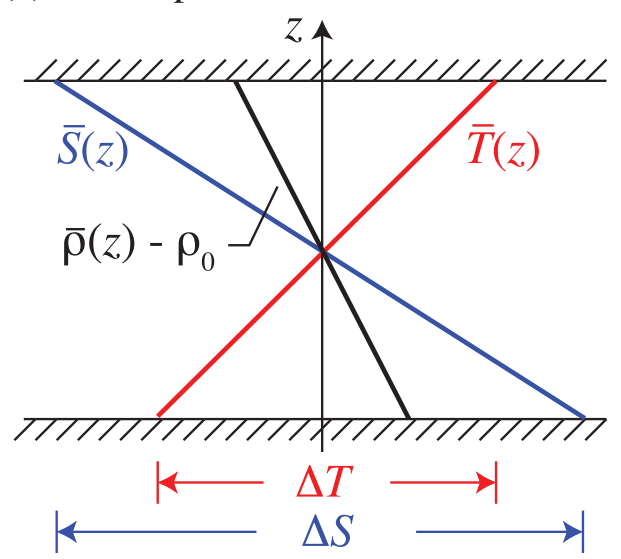

(b) Diffusive interface

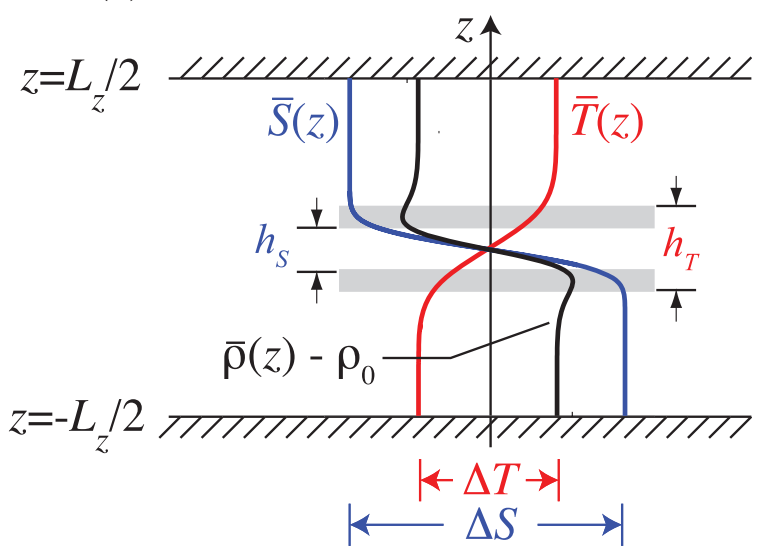

FIG. 1. (a) Linear profiles of the background $\bar{T}$ (red), $\bar{S}$ (blue), and $\bar{\rho}$ (black) for the analysis of DD instability. Both $T$ and $S$ are plotted in density units. (b) Profiles of a diffusive interface showing the relevant scales used in the analysis to follow. Gravitationally unstable boundary layers are visible in the density profile above and below the interface center. The length scale used to characterize these boundary layers is $b=\left(h_{T}-h_{S}\right) / 2$, indicated by the vertical thickness of the gray bars.

overlying warm, salty water, as shown in Fig. 1b (for a recent examination of the salt-finger interface, see Smyth and Kimura 2007). We show that the presence of the staircase considerably modifies the linear stability properties of the water column from the linear representation. Whereas the stability analysis of the linear $T-S$ profiles has been crucial in our understanding of the basic mechanism of instability, and recently in the process of staircase formation (Noguchi and Niino 2010), the stability analysis of the diffusive interface constitutes an important step in understanding the processes governing staircase evolution and maintenance. To our knowledge, the linear stability properties of the diffusive interface have not been studied previously.

Despite the clear mechanism of instability that has developed from analysis of the linear profiles, which consists of an exponentially growing oscillation of the water column, a different mechanism is often invoked for assessing the stability of a diffusive interface (Linden and Shirtcliffe 1978; Newell 1984; Padman and Dillon 1987, 1989; Worster 2004). In this case, the basic instability mechanism is thought to result from the formation of gravitationally unstable layers on either side of the interface (Fig. 1b). Because the $T$ interface diffuses more rapidly than the $S$ interface, molecular diffusion will tend to produce a relatively thicker $T$ interface (i.e., $h_{T}>h_{S}$; Fig. 1b). Because the $T$ profile is gravitationally unstable, this leads to a density profile that exhibits regions of gravitationally unstable fluid, which we will refer to as the diffusive boundary layer. It is the convective-type (nonoscillatory) instability of this boundary layer that is thought to be responsible for the maintenance of turbulence and enhanced mixing that occurs within the mixed layers of a thermohaline staircase. In keeping with the purely convective-type breakdown of the boundary layer, it has been assumed that a critical Rayleigh number that is characteristic of the boundary layer, of the order of $10^{3}$, must be exceeded for the boundary layers to break away from the interface (Linden and Shirtcliffe 1978).

The stability properties of the diffusive interface, as opposed to the linear profiles, are fundamental to understanding the enhanced fluxes of heat and salt present in thermohaline staircases. The microstructure profiles of $T$ and $S$ taken in the thermohaline staircase of Lake Kivu by T. Sommer et al. (2012, unpublished manuscript) clearly illustrate the presence of diffusive boundary layers (an example is shown in Fig. 2). The present study is largely motivated by these observations and the need to provide a more relevant theoretical understanding of the conditions limiting the growth of the boundary layer. The results of this study provide a basis for developing simple phenomenological models for DC (Linden and Shirtcliffe 1978; Newell 1984; Fernando 1989; Kelley 1990; Worster 2004), as well as in the interpretation of laboratory (Shirtcliffe 1973; Marmorino and Caldwell 1976; Newell 1984; Fernando 1989) and field measurements of the diffusive interface and its stability (Padman and Dillon 1987, 1989; Sánchez and Roget 2007; T. Sommer et al. 2012, unpublished manuscript).

After formulating the basic linear stability problem and its solution in section 2, we present the results of the stability analysis (section 3 ). These results shed light on the assumption of a purely convective-type instability of the boundary layer. In particular, we find that the first mode to become unstable is oscillatory and is more akin to the DC modes found in linear gradients than to a 


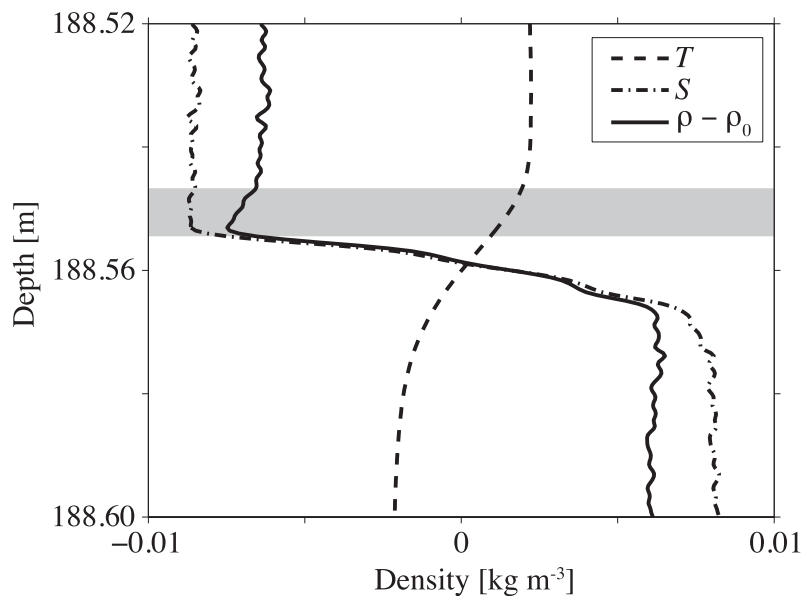

FIG. 2. Example of interface profiles measured with a highresolution microstructure profiler in the DD thermohaline staircase in Lake Kivu (for details of the measurements, see Sommer et al. 2012). The $T, S$, and $\rho$ profiles are plotted in density units, as in Fig. 1. A gravitationally unstable boundary layer in the $\rho$ profile, shown by the gray stripe, can be seen on the upper side of the $T$ and $S$ interfaces, which have different thicknesses.

purely convective mode. However, the assumption of steady $T$ and $S$ profiles that is used in the linear analysis is then tested in section 4 using direct numerical simulations (DNS) of the interface breakdown. The results of the DNS show that time-dependent effects are important for the type of unstable mode that develops (i.e., DC or convective), and the conditions under which the nonlinear breakdown of the interface takes place can exceed the linear predictions. By accounting for this diffusive growth of the interface in time, we propose that the dominant mode of instability for the diffusive interface is in fact of the convective type and is concentrated in the boundary layers. In section 5, we examine the applicability of a critical boundary layer Rayleigh number to describe the onset of instability and breakdown. Conclusions are stated in the final section.

\section{Formulation and solution of the linear stability problem}

\section{a. Formulation}

In this section, we derive the equations that govern the evolution of small (linear) perturbations to the background profiles (indicated with an overbar) of temperature $\bar{T}(z)$ and salinity $\bar{S}(z)$. We choose to represent $T$ and $S$ in density units so that the resulting density can be approximated by the following linear equation of state:

$$
\rho=\rho_{0}+T+S
$$

where $\rho_{0}$ is a reference density.
Our starting point is the two-dimensional incompressible Boussinesq equations of motion for a density-stratified fluid,

$$
\begin{aligned}
u_{t}+u u_{x}+w u_{z} & =-p_{x} / \rho_{0}+\nu \nabla^{2} u \\
w_{t}+u w_{x}+w w_{z} & =-p_{z} / \rho_{0}-g \rho / \rho_{0}+\nu \nabla^{2} w, \\
u_{x}+w_{z} & =0
\end{aligned}
$$

and we combine these with advection-diffusion equations for $T$ and $S$,

$$
\begin{aligned}
T_{t}+u T_{x}+w T_{z} & =\kappa_{T} \nabla^{2} T \text { and } \\
S_{t}+u S_{x}+w S_{z} & =\kappa_{S} \nabla^{2} S,
\end{aligned}
$$

and the equation of state (1). In the above equations we have represented partial derivatives by subscripts, where time is given by $t$; the horizontal and vertical coordinates are given by $(x, z)$ with velocity components of $(u, w)$, respectively; $p$ is the pressure; $\nu$ is the kinematic viscosity; and $\kappa_{T}$ and $\kappa_{S}$ the molecular diffusivities of $T$ and $S$, respectively. Now consider perturbations about a background state of no motion (i.e., $\bar{u}=\bar{w}=0$ ). We can write

$$
\begin{aligned}
T & =\bar{T}+\tilde{T}, \quad S=\bar{S}+\tilde{S}, \quad \rho=\bar{\rho}+\tilde{\rho}, \quad u=\tilde{u}, \\
w & =\tilde{w}, \quad \text { and } \quad p=\bar{p}+\tilde{p},
\end{aligned}
$$

where the tilde indicates perturbations from the background profiles, with the background pressure defined by hydrostatic balance. The background density profile is $\bar{\rho}(z)=\rho_{0}+\bar{T}(z)+\bar{S}(z)$.

We proceed to linearize the equations by substituting the decomposition in (4) and neglecting the products of the perturbation quantities, which is justified as long as they are small quantities. Solutions are then taken to be of the normal mode form with

$$
\{\tilde{w}, \tilde{T}, \tilde{S}\}=\{\hat{w}(z), \hat{T}(z), \hat{S}(z)\} e^{i k x+\sigma t}
$$

and similar expressions governing the other perturbation quantities. Here, $k$ is a horizontal wavenumber, which we take to be real; $\sigma=\sigma_{r}+i \sigma_{i}$ is a complex number, which is composed of a growth rate $\sigma_{r}$ and a frequency $\sigma_{i}$; and the hat denotes the vertical structure of the perturbation quantity. Choosing the normal mode form in (5) allows us to separate the $x$ and $t$ dependence and produces a set of ordinary differential equations in 
$z$. After eliminating two of the five equations and rearranging, we are left with the following set of three equations in the unknowns $\{\hat{w}, \hat{T}, \hat{S}\}$, which are all functions of $z$ only:

$$
\begin{aligned}
\sigma \hat{\nabla}^{2} \hat{w} & =\frac{k^{2} g}{\rho_{0}}(\hat{T}+\hat{S})+\nu \hat{\nabla}^{4} \hat{w}, \\
\sigma \hat{T} & =-\hat{w} \bar{T}^{\prime}+\kappa_{T} \hat{\nabla}^{2} \hat{T}, \quad \text { and } \\
\sigma \hat{S} & =-\hat{w} \bar{S}^{\prime}+\kappa_{S} \hat{\nabla}^{2} \hat{S},
\end{aligned}
$$

where $\hat{\nabla}^{2} \equiv d^{2} / d z^{2}-k^{2}$ and primes denote ordinary differentiation with respect to $z$.

It is important to note that in arriving at this system of equations we have made a quasi-steady assumption for the evolution of the background profiles. This is equivalent to neglecting the molecular diffusion of these profiles in time and is necessary in order to carry out a standard stability analysis. This assumption is not necessary for the stability of the linear profiles (because they are steady solutions to the diffusion equation), and we will test the accuracy of this assumption using the simulations. In addition, we have limited the analysis to two dimensions only for simplicity. The laboratory experiment discussed in Linden and Shirtcliffe (1978) indicates that the instabilities at the diffusive interface can take three-dimensional forms, and a more complete analysis must be left for future study.

The equations in (6) describe an eigenvalue problem for the eigenvalue $\sigma$ and the eigenfunctions $\{\hat{w}, \hat{T}, \hat{S}\}$. It is generally the case that there may be more than one value of $\sigma$ and the associated eigenfunctions, for a single value of $k$, and each of these solutions will be referred to as a mode. The stability is determined entirely by the growth rate $\sigma_{r}$; if $\sigma_{r}>0$ the perturbations will grow in time, whereas for $\sigma_{r}<0$ they will decay in time. As they grow or decay, the modes may also oscillate with frequency $\sigma_{i}$. In addition, the vertical structure of the perturbations is described by the eigenfunctions $\{\hat{w}, \hat{T}, \hat{S}\}$, which are functions of $z$.

Before discussing the solution procedure of these equations, it is convenient to first nondimensionalize them using the following scales: length scale $L$, velocity scale $\kappa_{T} / L$, time scale $L_{2} / \kappa_{T}, T$ density scale $\Delta T$, and $S$ density scale $\Delta S$, where $L$ is left (for now) as an arbitrary length scale and the density differences due to $T$ and $S$ are defined in Fig. 1. For dimensionless quantities, we use the following definitions:

$$
\begin{aligned}
\zeta & =\frac{z}{L}, \quad \sigma_{*}=\sigma \frac{L^{2}}{\kappa_{T}}, \quad \hat{w}_{*}=\hat{w} \frac{L}{\kappa_{T}}, \quad \alpha=k L, \\
\hat{\nabla}_{*}^{2} & =\hat{\nabla}^{2} L^{2}, \quad \hat{T}_{*}=\hat{T} / \Delta T, \quad \text { and } \quad \hat{S}_{*}=\hat{S} / \Delta S .
\end{aligned}
$$

Then the nondimensional equations can be written as

$$
\begin{aligned}
& \sigma_{*} \hat{\nabla}_{*}^{2} \hat{w}_{*}= \operatorname{Ra}_{L} \operatorname{Pr} \alpha^{2}\left(\hat{T}_{*}+R \hat{S}_{\text {S }}\right)+\operatorname{Pr} \hat{\nabla}_{*}^{4} \hat{w}_{*}, \\
& \sigma_{*} \hat{T}_{*}=-\hat{w}_{*} \bar{T}_{*}^{\prime}+\hat{\nabla}_{*}^{2} \hat{T}_{*}, \quad \text { and } \\
& \sigma_{*} \hat{S}_{*}=-\hat{w}_{*} \bar{S}_{*}^{\prime}+\tau \hat{\nabla}_{*}^{2} \hat{S}_{*},
\end{aligned}
$$

where primes now indicate differentiation with respect to $\zeta$ and we have defined the following important dimensionless numbers:

$\mathrm{Ra}_{L} \equiv \frac{g \Delta T L^{3}}{\rho_{0} \nu \kappa_{T}}, \quad R_{\rho} \equiv \frac{\Delta S}{\Delta T}, \quad \operatorname{Pr} \equiv \frac{\nu}{\kappa_{T}}, \quad$ and $\quad \tau \equiv \frac{\kappa_{S}}{\kappa_{T}}$.

These correspond to the well-known Rayleigh number, density ratio, Prandtl, and diffusivity ratio, respectively. In the case of the Rayleigh number, we shall use the $L$ subscript to explicitly indicate which of three possible types of Rayleigh number is referred to, each with a different length scale $L$ and possibly a different density scale. Both $\operatorname{Pr}$ and $\tau$ are properties of the fluid and the diffusing scalars, and herein we will restrict ourselves to heat and salt in water that is $25^{\circ} \mathrm{C}$, representative of Lake Kivu (Newman 1976; Schmid et al. 2010). This results in $\operatorname{Pr} \approx 6$ and $\tau \approx 0.01$, which will be used throughout the paper. In addition, we will drop the asterisk notation hereafter and deal only with dimensionless quantities unless stated.

In nondimensional terms, the vertical boundaries extend from $\zeta=-H / 2$ to $\zeta=H / 2$, where $H \equiv L_{z} / L$ and $L$ remains to be defined for the particular problem of interest. The set of equations in (7) is subject to the following conditions on these boundaries:

(i) $\hat{w}=0$ (rigid lid),

(ii) $\hat{u}^{\prime}=0 \Rightarrow \hat{w}^{\prime \prime}=0$ (stress free), and

(iii) $\hat{T}=0$ and $\hat{S}=0($ constant $T$ and $S)$.

\section{b. Numerical solution method}

The set of equations in (7), governing the linear stability, is solved numerically for all $\bar{T}$ and $\bar{S}$ profiles except the linear profiles, which admit a straightforward analytical solution (see, e.g., Baines and Gill 1969; Turner 1973; Linden 2000). Similar to Smyth and Kimura (2007), we use a Galerkin method whereby a truncated series representation of the eigenfunctions is assumed: namely,

$$
\{\hat{w}(\zeta), \hat{T}(\zeta), \hat{S}(\zeta)\}=\sum_{n=1}^{N}\left\{\hat{w}_{n}, \hat{T}_{n}, \hat{S}_{n}\right\} f_{n}(\zeta)
$$


where $\left\{\hat{w}_{n}, \hat{T}_{n}, \hat{S}_{n}\right\}$ are coefficients and $f_{n}(\zeta)$ is chosen to satisfy the boundary conditions in each case. For those listed above, this corresponds to

$$
f_{n}(\zeta)=\sin \left[\frac{n \pi(\zeta+H / 2)}{H}\right]
$$

and the series is a truncated Fourier representation of the eigenfunctions. Similar to the standard procedure for determining the Fourier coefficients of a function, we multiply each equation in (7) by $f_{m}(\zeta)$ and take the inner product, defined by

$$
\langle\beta(\zeta)\rangle \equiv \frac{2}{H} \int_{0}^{H} \beta(\zeta) d \zeta
$$

where $\beta(\zeta)$ is some arbitrary function. Using the identity $\left\langle f_{n}(\zeta) f_{m}(\zeta)\right\rangle=\delta_{m n}$, where $\delta_{m n}$ is the Kroneker delta, we can write the system of equations as

$$
\begin{gathered}
\sigma \alpha_{m}^{2} \hat{w}_{m}=-\operatorname{RaPr} \alpha^{2}\left(\hat{T}_{m}+R_{\rho} \hat{S}_{m}\right)-\operatorname{Pr} \alpha_{m}^{4} \hat{w}_{m} \\
\sigma \hat{T}_{m}=-\sum_{n=1}^{N} \hat{w}_{n}\left\langle\bar{T}^{\prime}(\zeta) f_{n}(\zeta) f_{m}(\zeta)\right\rangle-\alpha_{m}^{2} \hat{T}_{m}, \quad \text { and } \\
\sigma \hat{S}_{m}=-\sum_{n=1}^{N} \hat{w}_{n}\left\langle\bar{S}^{\prime}(\zeta) f_{n}(\zeta) f_{m}(\zeta)\right\rangle-\tau \alpha_{m}^{2} \hat{S}_{m}
\end{gathered}
$$

where we define $\alpha_{n}^{2} \equiv(n \pi / H)^{2}+\alpha^{2}$ for a more compact notation. The equations in (11) have the form of a matrix eigenvalue problem $\mathbf{A X}=\sigma \mathbf{X}$, with $\mathbf{X}$ a vector of size $3 N$ that is formed by concatenating all of the Fourier coefficients $\left\{\hat{w}_{n}, \hat{T}_{n}, \hat{S}_{n}\right\}$ together into a single vector. This form facilitates the solution of the problem using standard matrix eigenvalue software (in our case, the $\mathrm{R}$ software package was used).

\section{Linear stability properties}

In this section, we present the results of the stability analysis that was formulated in the previous section. Before discussing new results for the diffusive interface, we shall briefly review some important results from the analysis of the linear profiles.

\section{a. Linear profiles}

Following the previous work of Stern (1960), Veronis (1965), Nield (1967), and Baines and Gill (1969) (see also Turner 1973; Linden 2000), we take linear background profiles for $\bar{T}$ and $\bar{S}$,

$$
\bar{T}(\zeta)=\zeta \quad \text { and } \quad \bar{S}(\zeta)=-\zeta
$$

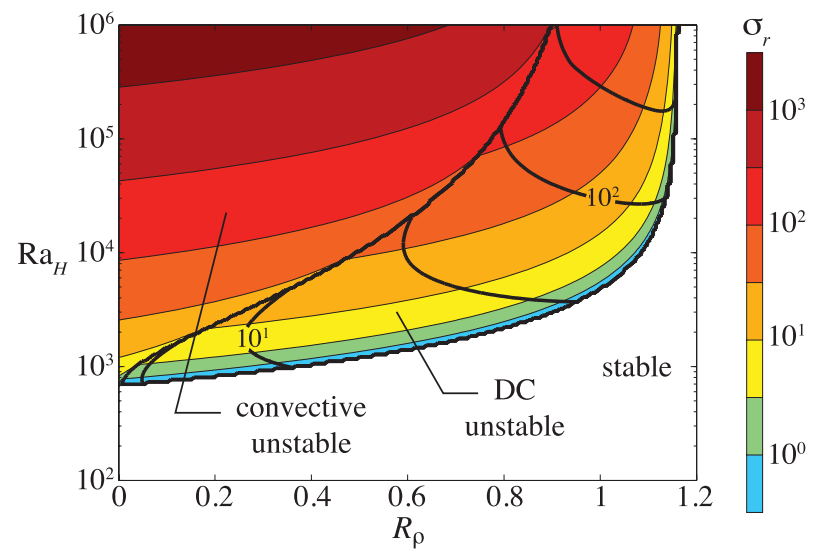

FIG. 3. Stability diagram of the classical linear profiles. Colored contours are of the growth rate $\sigma_{r}$ on the logarithmic scale shown, whereas the thick dark contour lines are of frequency $\sigma_{i}$, which are also on a logarithmic scale. The DC unstable modes with $\sigma_{i}>$ 0 occur in the region with thick dark contour lines, and convectivetype unstable modes are found in the higher $\mathrm{Ra}_{H}$ and $R_{\rho}<1$ region as labeled. No instability is present when $R_{\rho}>1.16$, no matter how large $\mathrm{Ra}_{H}$.

on the domain $-1 / 2 \leq \zeta \leq 1 / 2$. This is equivalent to choosing the domain height $L_{z}$ as the length scale to nondimensionalize by (the only available extrinsic length scale). The dimensionless domain height is unity, and the appropriate Rayleigh number is

$$
\mathrm{Ra}_{H} \equiv \frac{g \Delta T L_{z}^{3}}{\rho_{0} \nu \kappa_{T}} .
$$

The linear profiles in (12) considerably simplify the system of equations in (7), and an analytical solution is possible. The dispersion relation governing the eigenvalues can be found in Baines and Gill (1969), and the reader is referred to this work as well as Turner (1973) and Linden (2000) for further details.

The results of the stability analysis are most conveniently displayed on what we shall refer to as a stability diagram, which is shown in Fig. 3. It contours the $R_{\rho}-\mathrm{Ra}_{H}$ plane with the positive unstable growth rates $\sigma_{r}$ in thin contours and color filling and the frequency of oscillation $\sigma_{i}$ in thick contours. At each point in the unstable region (i.e., for a fixed $\mathrm{Ra}_{H}$ and $R_{\rho}$ ), there is generally a continuous band of unstable wavenumbers present. However, we plot only the mode with the largest growth rate, because it is this "most unstable mode" that is expected to dominate the initial linear growth phase and possibly the nonlinear transition to turbulent convection.

Three different regions of the diagram in Fig. 3 may be identified:

(i) A stable region exists for either sufficiently low $\mathrm{Ra}_{H}$ or sufficiently high $R_{\rho}$. The lowest $\mathrm{Ra}_{H}$ that may be 
unstable occurs for $R_{\rho}=0$, in which no gravitationally stabilizing $S$ stratification is present, and we recover the classical case of pure thermal convection in a linear gradient (Linden 2000; Kundu et al. 2004). This has the well-known solution that instability is triggered once a critical $\mathrm{Ra}_{H, \mathrm{cr}}=27 \pi^{4} / 4=$ 658 is exceeded (though this value depends on the form of the boundary conditions). The resulting unstable modes are of the convective type and form the second region of the diagram.

(ii) In the large $\mathrm{Ra}_{H}$, small $R_{\rho}$ portion of the diagram, instability is due to a convective-type instability; the unstable modes are nonoscillatory with $\sigma_{i}=0$. The physical mechanism of growth for this convective type of instability is straightforward: the perturbations are able to extract the potential energy available in the gravitationally unstable (top heavy) density field to directly increase their amplitude over time, once the stabilizing effects of viscosity and diffusion are overcome. These convective-type unstable modes exist only when the density field is gravitationally unstable and so are limited to the region $0 \leq R_{\rho}<1$, assuming that $\mathrm{Ra}_{H}$ is sufficiently large.

(iii) The third portion of the diagram consists of DCtype unstable modes that have an oscillating growth with $\sigma_{i}>0$ (region of thick dark contour lines in Fig. 3). These DC-type modes are the first to become unstable as $\mathrm{Ra}_{H}$ is increased and are the only type of instability that is possible when the density stratification is gravitationally stable (i.e., $R_{\rho}>1$ ). The physical mechanism of instability can be understood as follows: After any small displacement from its equilibrium level, a fluid parcel will find itself in a different $T-S$ state than it originated from. Because of the differing rates of molecular diffusion, the parcel exchanges $T$ more rapidly than $S$ with its surrounding environment. Because $S$ is stably stratified, the parcel either sinks or rises back to its original level, but it overshoots, because it carries with it a density anomaly. The density anomaly grows because the parcel is now exchanging $T$ in more or less buoyant surroundings. The oscillation can therefore grow in time. However, the DC instability is very restricted in that it cannot operate if $R_{\rho}$ is too large, and the flow is found to be stable for $R_{\rho}>$ $(\operatorname{Pr}+1) /(\operatorname{Pr}+\tau) \approx 1+\operatorname{Pr}^{-1} \approx 1.16$. Also, the transition between the DC-type modes and the convective-type modes occurs at $R_{\rho} \rightarrow 1$ as $\mathrm{Ra}_{H} \rightarrow \infty$.

Because most geophysical observations of DC unstable profiles have a mean $R_{\rho}>1.16$ (Padman and Dillon 1987; Kelley et al. 2003; Schmid et al. 2004;
Timmermans et al. 2008; Schmid et al. 2010), it is unlikely that the instability of the linear profiles plays a role in these systems. Therefore, in order to assess DC processes in thermohaline staircases, we now turn to the stability of the diffusive interface.

\section{b. The diffusive interface}

When considering the stability of a diffusive interface, it is necessary to introduce two additional length scales into the problem (in addition to the vertical domain size $L_{z}$ ). These are the thicknesses of both the $\bar{T}$ and $\bar{S}$ interfaces, which will be denoted by $h_{T}$ and $h_{S}$, respectively (Fig. 1b). Each interfacial thickness is defined by the dimensional relation

$$
h_{\varphi} \equiv \Delta \varphi /\left(\frac{\partial \varphi}{\partial z}\right)_{\varphi_{0}},
$$

where $\varphi$ represents either $T$ or $S$ and the $\varphi_{0}$ subscript indicates that the derivative is evaluated on the $T-S$ scalar value at the interface center. In the case of the simulations, we calculate the gradient by averaging over $-\Delta \varphi / 8<\varphi_{0}<\Delta \varphi / 8$.

In our analysis of the diffusive interface we shall choose $L=h_{T}$ as the length scale in the nondimensionalization. Two different model profiles will be considered, (i) the "erf" interface model with

$$
\bar{T}(\zeta)=\operatorname{erf}(\sqrt{\pi} \zeta) / 2 \quad \text { and } \quad \bar{S}(\zeta)=-\operatorname{erf}(\sqrt{\pi} r \zeta) / 2
$$

and (ii) the "tanh" interface model with

$$
\bar{T}(\zeta)=\tanh (2 \zeta) / 2 \quad \text { and } \quad \bar{S}(\zeta)=-\tanh (2 r \zeta) / 2
$$

which are both nondimensional. Note that the $\sqrt{\pi}$ factor and the factor of 2 appear in the arguments in order to satisfy (14). In Fig. 1b, the difference between the erf and the tanh profiles would be virtually indistinguishable. However, these two different profiles are used to assess the sensitivity of the results; the erf interface is a solution to the diffusion equation, whereas the tanh interface is an analytically convenient form.

With the introduction of the $h_{T}$ and $h_{S}$ scales, come two additional dimensionless numbers,

$$
r \equiv h_{T} / h_{S} \quad \text { and } \quad H \equiv L_{z} / h_{T}
$$

the interfacial thickness ratio and the dimensionless domain height, respectively. When examining the diffusive interface, $H$ measures the relative thickness of the interface to the mixed layers above and below. Within thermohaline staircases, the interfaces are generally found 


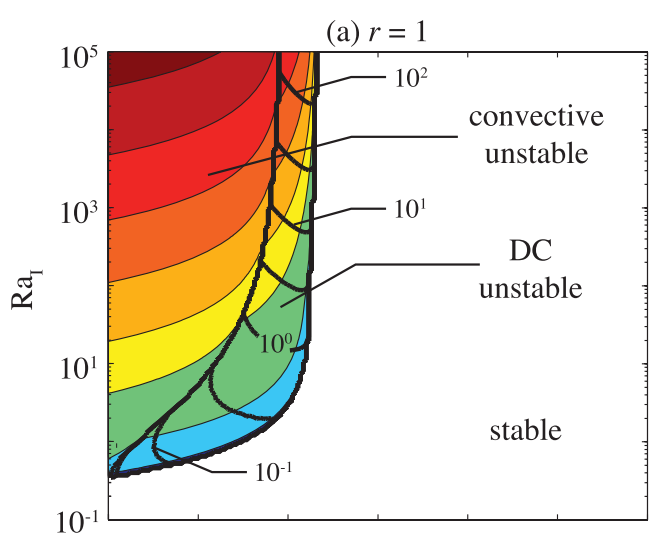

(c) $r=1.5$

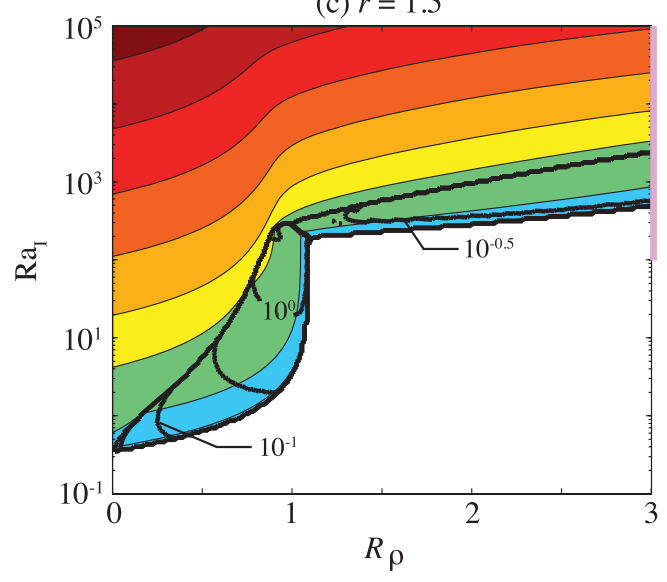

(b) $r=1.25$

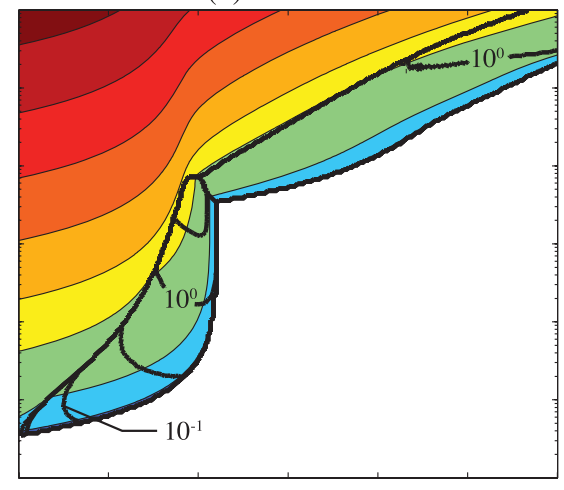

(d) $r=2$

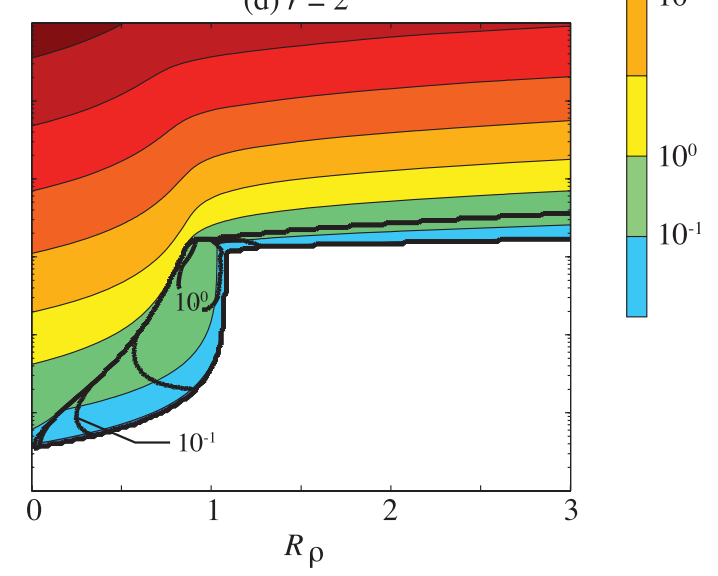

FIG. 4. Stability properties of the diffusive interface at various $r$ for the tanh interface model with $H=L_{z} / h_{T}=10$, $\operatorname{Pr}=6$, and $\tau=0.01$. All notations as in Fig. 3. (c) The vertical pink strip indicates the location of the plot in Fig. 5 .

to be much thinner than the mixed layers on either side (Neal et al. 1969; Turner 1973; Padman and Dillon 1989; Schmid et al. 2010), and so we shall generally restrict ourselves to $5 \leq H \leq 40$. In addition, we will treat $r$ as an independent variable in the analysis that follows; however, it is likely that it is controlled by the physics of diffusive convection. If it is pure molecular diffusion that is acting within the interfaces, then an upper bound is found to be $r \leq \tau^{-1 / 2} \approx 10$, whereas a reasonable lower bound would be $r=1$. We shall therefore restrict ourselves to this range $1 \leq r \leq 10$, which is also the range that has been reported in the laboratory experiments of Marmorino and Caldwell (1976).

It is also more appropriate, when examining the stability of the diffusive interface, to use an interfacial length scale in defining the Rayleigh number, and so we shall use

$$
\mathrm{Ra}_{I} \equiv \frac{g \Delta T h_{T}^{3}}{\rho_{0} \nu \kappa_{T}}
$$

as the "interfacial Rayleigh number."
Stability diagrams using the tanh interface model are shown in Fig. 4 for various $r$. Beginning with $r=1$ (Fig. 4a), the profiles of $T$ and $S$ have the same interface thicknesses. In this case, no boundary layers exist, and for $R_{\rho}>1$ the background density field is gravitationally stable for all $\zeta$. We should intuitively expect that the stability diagram show similar behavior to the linear gradient results in Fig. 3. This is because approximately linear gradients are present within the interfaces and only the length scale has changed, along with the proximity of the vertical boundaries. In comparing Figs. 4a and 3, this is indeed what is observed. In both cases, there is a stable region at low $\mathrm{Ra}$, and the cutoff in $R_{\rho}$ occurs at the same value of 1.16, above which the profiles are stable.

As $r$ is increased from unity, diffusive boundary layers develop above and below the gravitationally stable interface center (Fig. 1b). These boundary layers can be seen to modify the stability properties by destabilizing the profiles at larger $R_{\rho}$ values. As is to be expected, the larger $r$ becomes-and therefore the thicker and more gravitationally unstable the boundary layers becomethe lower is the $\mathrm{Ra}_{I}$ required to achieve instability. 


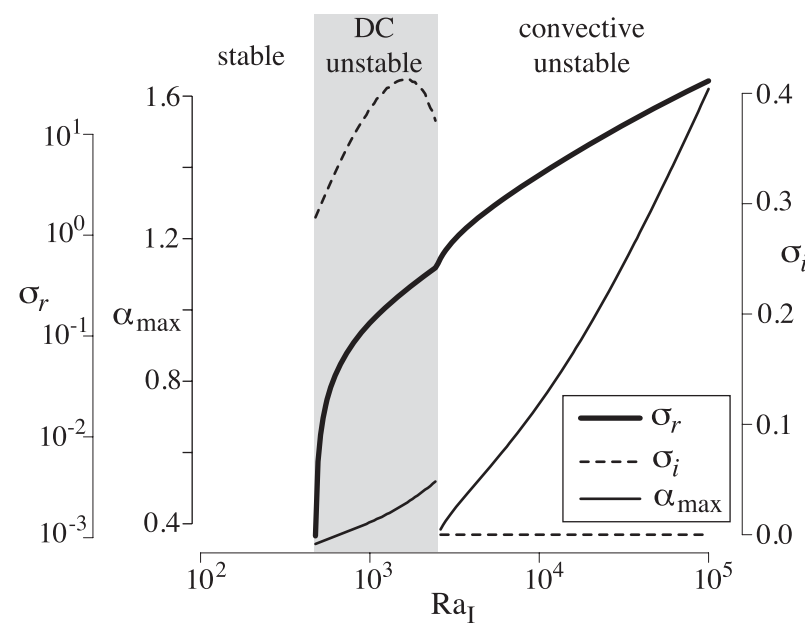

FIG. 5. Cut along $R_{\rho}=3.0$ for varying $\mathrm{Ra}_{\mathrm{I}}$ from Fig. $4 \mathrm{c}$ with $r=$ 1.5 showing $\sigma_{r}, \sigma_{i}$, and $\alpha_{\text {max }}$. The three distinct regions of stable, DC unstable, and convective unstable modes can clearly be seen with a jump in $\alpha_{\max }$ and $\sigma_{i}$.

Despite the changes that occur in the stability properties once diffusive boundary layers are present (i.e., for $r>$ 1), each of the panels in Fig. 4 shows a transition from stability to DC-type unstable modes to convective-type unstable modes as $\mathrm{Ra}_{I}$ is increased. The DC-type modes are always found to be adjacent to the stability boundary; however, the width of the DC-type unstable region is found to shrink as $r$ increases, and the boundary layers grow in size and strength. At $r=10$ (not shown), the expected upper limit, the DC-type unstable region is still present but confined to a very small region close to the stability boundary.

To emphasize the distinction between the convectivetype modes and the DC-type unstable modes, we plot in Fig. 5 a cut along $R_{\rho}=3$ while varying $\mathrm{Ra}_{I}$. This represents the cut in Fig. 4c indicated in pink with $r=1.5$. The plot shows the growth rate $\sigma_{r}$, frequency $\sigma_{i}$, and the wavenumber of maximum growth $\alpha_{\max }$ for the most amplified mode. A jump in $\alpha_{\max }$ and $\sigma_{i}$ are present at the transition between the DC- and convective-type unstable modes, as well as a change in the trend of $\sigma_{r}$. It should be noted that the transition from a DC- to convective-type unstable mode occurs because the growth rate of the convective mode becomes larger than that of the DC mode, not because the mode ceases to become unstable.

Another feature that is apparent in Fig. 4 is the similarity, in all panels, of the $R_{\rho}<1$ portion of the diagrams. This suggests that, in this region, the stability of the profiles is governed by the entire interface, opposed to the boundary layers at larger $R_{\rho}$, because no boundary layers are present for $r=1$. This behavior can be seen by comparing the eigenfunctions from these regions of the diagram because the eigenfunctions give the vertical structure of the unstable mode. In Fig. 6, we fix $r=1.25$ and $\mathrm{Ra}_{I}=10^{4}$ but vary $R_{\rho}$ in order to cross from an interface-dominated instability at $R_{\rho}=0.5$ to boundary layer instabilities at $R_{\rho}=1.5$. This can be seen in the amplitudes of both the vertical velocity and temperature perturbation eigenfunctions, $|\hat{w}|$ and $|\hat{T}|$. The interfacecentered modes exhibit maximum amplitudes at the interface level (Fig. 6b), whereas the boundary layer modes display maximums above and below the interface (Fig. 6c). The position of the $|\hat{T}|$ maximum is the vertical location the instability will appear from when viewing the $T$ field.

We now compare these results for the tanh interface to the same analysis using the erf interface model. Although the two different models show the same qualitative behavior, there are differences in the location of the stability boundary. As an example, we plot the $\mathrm{Ra}_{I}$ at marginal stability for two fixed values of $R_{\rho}$ for various $r$ in Fig. 7. This shows that the exact form of the profiles is important in determining the stability boundary, particularly when $r$ is close to unity. However, the stability diagrams (as in Fig. 4) show the same structure in each case, with both interface-centered and boundary layercentered regions and DC unstable modes composing the stability boundary.

\section{Simulating interface instability}

In our linear stability analysis of the diffusive interface in the previous section we identified two principle modes of instability: the oscillating DC type and the purely convective type. Furthermore, depending primarily on the density ratio $R_{\rho}$, these modes can grow from within the interface or the boundary layers. In this section, we test these predictions using DNS. In particular, the DNS allows us to examine the instability of diffusive interfaces that are growing in time due to molecular diffusion. This time dependence was not explicitly accounted for in the linear stability analysis. A central question that we pursue is whether the boundary layer DC unstable modes that compose the stability boundary at $r>1$ are significant in the breakdown of the boundary layers when a time-dependent interface is considered.

\section{a. Description of the simulations}

The DNS is performed using the code originally described in Winters et al. (2004), which has been modified to carry a second scalar by Smyth et al. (2005). The second scalar field is resolved on a grid that has a spacing twice as fine as the other fields and so is especially suitable to study the low $\tau$ that is present in the heat-salt system. The addition of the second scalar field, however, 
(a) profiles

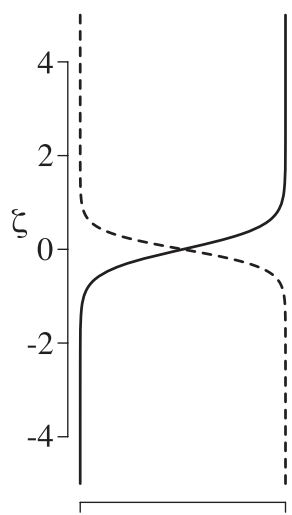

$\bar{S}, \bar{T}$ (b) $R_{\rho}=0.5$

(c) $R_{\rho}=1.5$

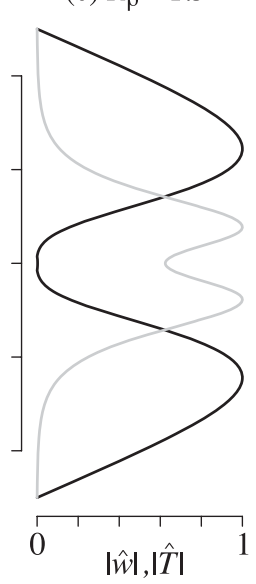

FIG. 6. (b),(c) Amplitudes of the eigenfunctions $|\hat{w}|$ (black curves) and $|\hat{T}|$ (gray curves) showing the presence of both interface-centered and boundary layer-centered modes at the $R_{\rho}$ indicated. (a) The $\bar{T}$ (solid line) and $\bar{S}$ (dashed line) profiles are shown for reference. Other parameters are $\mathrm{Ra}_{I}=10^{4}, r=1.25$, and $H=10$.

is restricted only to three-dimensional rectangular domains. We therefore choose a relatively small scale for the third dimension $L_{y}$-compared to the horizontal $L_{x}$ and vertical $L_{z}$ - scales in testing the two-dimensional linear predictions. All of the parameters chosen in the DNS are summarized in Table 1.

Of the five simulations that were performed, four are used to directly test the linear predictions by "turning off" the molecular diffusion of the background $T-S$ profiles (simulations I-IV in Table 1). In each of these cases, which will be referred to as "stationary," there is a well-defined $\sigma$ and $\alpha_{\max }$ that can be compared with the linear predictions. It is also of interest to observe the finite-amplitude motions of these instabilities before proceeding to the time-dependent nonstationary case where molecular diffusion is acting on the background profiles.

Each simulation is initialized with background $\bar{T}$ and $\bar{S}$ profiles, where the corresponding dimensionless parameters $\mathrm{Ra}_{I}, R_{\rho}, r, \operatorname{Pr}, \tau$, and $H$ are chosen. In the stationary cases, these parameters do not change in time; however, in the nonstationary case, $\mathrm{Ra}_{I}, r$, and $H$ are all functions of $t$. All simulations are also initialized by random noise in the velocity field that is centered on the interface level. This random perturbation is a seed for the instability to grow from. The boundary conditions are periodic in the horizontal directions $(x, y)$ and correspond to a free-slip, rigid-lid condition on the velocity field and a no-flux condition in the $T-S$ fields on the vertical boundaries. Although the linear stability analysis used a constant $T-S$ condition on the vertical boundaries, it is

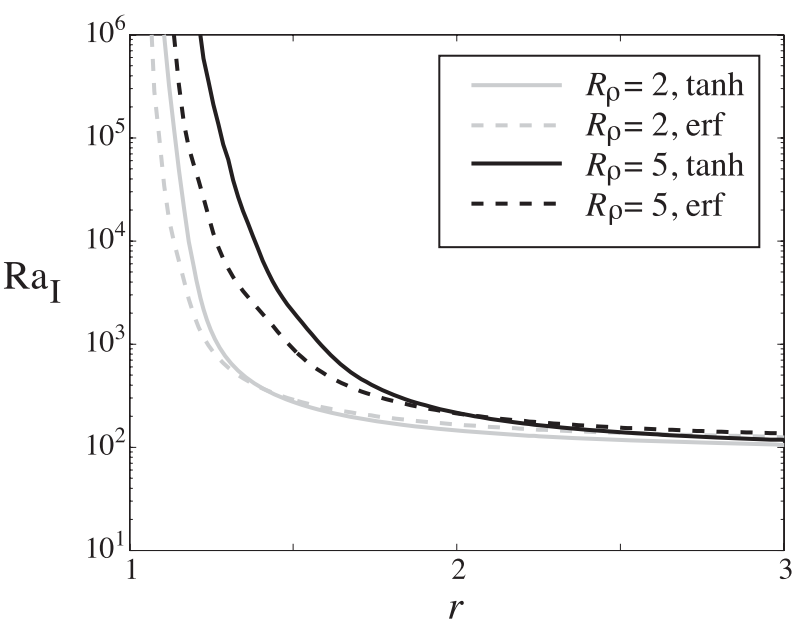

FIG. 7. Location of the stability boundary in the $r-\mathrm{Ra}_{\mathrm{I}}$ plane for $R_{\rho}=2$ and 5, comparing the tanh interface with the erf interface.

not expected to significantly affect the results, and this will be confirmed in the following.

\section{b. Testing the linear predictions: The stationary interface}

As an initial test of the linear analysis, we have chosen four simulations (I-IV in Table 1) that are predicted to produce a stable mode (I), a DC-type unstable boundary layer mode (II), a convective-type unstable boundary layer mode (III), and a DC-type unstable interface mode (IV).

The growth of the perturbations in time is best seen by plotting the volume-averaged kinetic energy. In the case of simulation I, the kinetic energy simply decayed in time (not shown). The other simulations, II-IV, are shown in Figs. 8a-c, respectively. Because the vertical axis is a logarithmic scale, a strictly exponentially growing perturbation will appear as a straight line, as is predicted by the linear theory. This growth rate prediction (dashed lines) is found to be in agreement with the DNS results, despite the fact that the growth rates each differ by nearly an order of magnitude in each case. Oscillations in the kinetic energy are observed only in the DC-type unstable modes (simulations II and IV in Figs. 8a,c), and the predicted period of oscillation, given by the scale indicated by the arrows, is also in agreement with the simulations.

It is also possible to test the linear predictions of whether the instability will be centered in the boundary layers or the interface. This can be seen in Figs. 9a,b, where contours of the $T$ field are shown for simulations III and IV once the instability has developed a finite amplitude. In the case of Fig. 9a, the central core of the interface is left virtually undisturbed, and the displacements are focused in the boundary layers at the interface 
TABLE 1. Values of various important parameters for the simulations performed. The gridpoint resolution listed for the simulations $\left\{N_{x}, N_{y}, N_{z}\right\}$ is that of the $S$ field. The $T$, velocity, and pressure fields are resolved on a grid with half this resolution. In the case of the nonstationary simulation $(\mathrm{V})$, the dimensionless parameters listed are evaluated using the initial conditions. In all cases, $\operatorname{Pr}=6, \tau=0.01$, and $L_{y} / h_{T}=0.66$. Also included is the total number of waves (in the horizontal $x$ direction) predicted in the domain $n$. Here, $L_{x}$ and $N_{x}$ have been doubled for simulation II to ensure two wavelengths of the most unstable mode are possible. Note that the value of $H$ in these simulations is different from that of Fig. 4, and they cannot be directly compared.

\begin{tabular}{lcccccrr}
\hline \hline & Simulation & $\left\{N_{x}, N_{y}, N_{z}\right\}$ & $\mathrm{Ra}_{I}$ & $R_{\rho}$ & $r$ & $H$ & $n$ \\
\hline I & Stationary & $\{360,16,180\}$ & $10^{3}$ & 3 & 1.5 & 8 & - \\
II & Stationary & $\{720,16,180\}$ & $1.5 \times 10^{3}$ & 3 & 1.5 & 8 & 2 \\
III & Stationary & $\{360,16,180\}$ & $10^{4}$ & 3 & 1.5 & 8 & 2 \\
IV & Stationary & $\{360,16,180\}$ & $2 \times 10^{5}$ & 1.1 & 1 & 8 & 13 \\
V & Nonstationary & $\{800,32,400\}$ & $10^{4}$ & 3 & 1 & 15 & - \\
\hline
\end{tabular}

edges. In contrast, the predicted DC interface-centered instability of Fig. 9b shows displacements that are focused within the interface itself. In this case, the interface performs exponentially growing standing oscillations.

Because it is necessary to choose a finite horizontal domain size $L_{x}$ in the DNS, it is not possible to represent a continuous range of $\alpha$. Instead, the periodic boundary conditions allow only discrete values of $\alpha=2 \pi n h_{T} / L_{x}$, where $n=1,2, \ldots$ is the number of waves in the domain. The wavenumber that is expected to emerge from the DNS is that with the largest growth rate, and this is not necessarily the wavenumber of maximum growth $\alpha_{\max }$. In Table 1, the total number of waves $n$ predicted by the linear theory is listed. For each of the $T$ fields plotted in Figs. 9a,b, the linear predictions agree with the number of waves observed. The wavelengths predicted by the theory, once corrected for the periodic boundary conditions, correspond to the length of the scales shown in Figs. 9a,b.

\section{c. Breakdown of the time-dependent diffusive interface}

In the nonstationary case, the simulation $(\mathrm{V})$ is begun with $T$ and $S$ interfaces that have an equal thickness $(r=1)$ and are allowed to diffuse in time. The initial $\mathrm{Ra}_{I}, R_{\rho}$, and $H$ are chosen such that the profiles are stable at all wavenumbers. Each interface will then grow in time due to molecular diffusion, according to

$$
h_{\varphi}(t)=2\left[\pi \kappa_{\varphi}\left(t+t_{\varphi}\right)\right]^{1 / 2}
$$

in the case of the erf interface. Here, $\varphi$ represents $T$ and $S$, and the $t_{\varphi}$ time shift is chosen to satisfy the initial interface thicknesses. The evolution of the linear stability properties of the diffusive interface may then be followed in the $r-\mathrm{Ra}_{I}$ plane, as shown in Fig. 10.

At the initial time $t=0$, the interface has a position on the diagram corresponding to the initial values of $r$ and $\mathrm{Ra}_{I}$ and is located in the stable region (Fig. 10). The position of this boundary, however, is changing in time because of its dependence on $H(t)$. At a later time, both $r$ and $\mathrm{Ra}_{I}$ have increased. The location of the interface therefore moves upward and rightward on the trajectory shown in Fig. 10, whereas the position of the stability boundary moves away as $H$ decreases. During the simulation the interface crosses into the unstable portion of the diagram, thus initiating instability. A complete breakdown of the boundary layers occurs at the final position shown in Fig. 10.
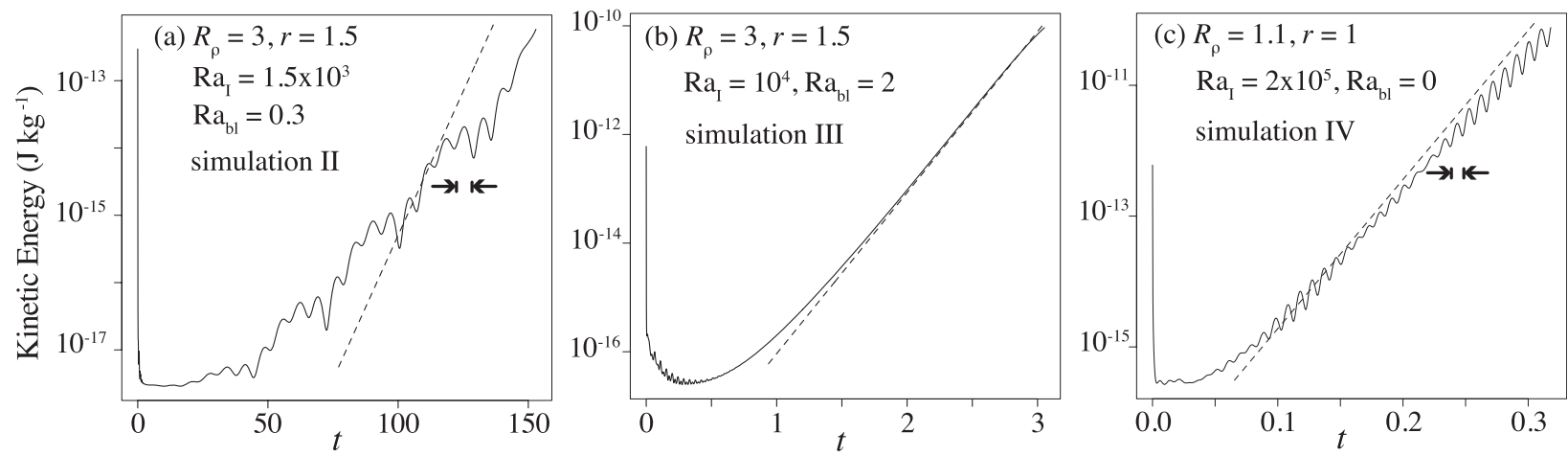

FIG. 8. Three simulations (II-IV) designed to test the linear predictions. These correspond to (a) a DC unstable boundary layer mode (II), (b) a convective unstable boundary layer mode (III), and (d) a DC unstable interface mode (IV). The time evolution of the volumeaveraged kinetic energy is shown by the solid lines. The slope of the curves is a measure of the growth rate $\sigma_{r}$ and can be compared with the predicted $\sigma_{r}$ from the linear theory shown as dashed lines. The time interval indicated by arrows in (a),(c) indicates the predicted oscillation period given by $4 \pi / \sigma_{i}$ (the kinetic energy has twice the oscillation frequency of other fields). A time scale of $h_{T}{ }^{2} / \kappa_{T}$ has been used to nondimensionalize $t$. 

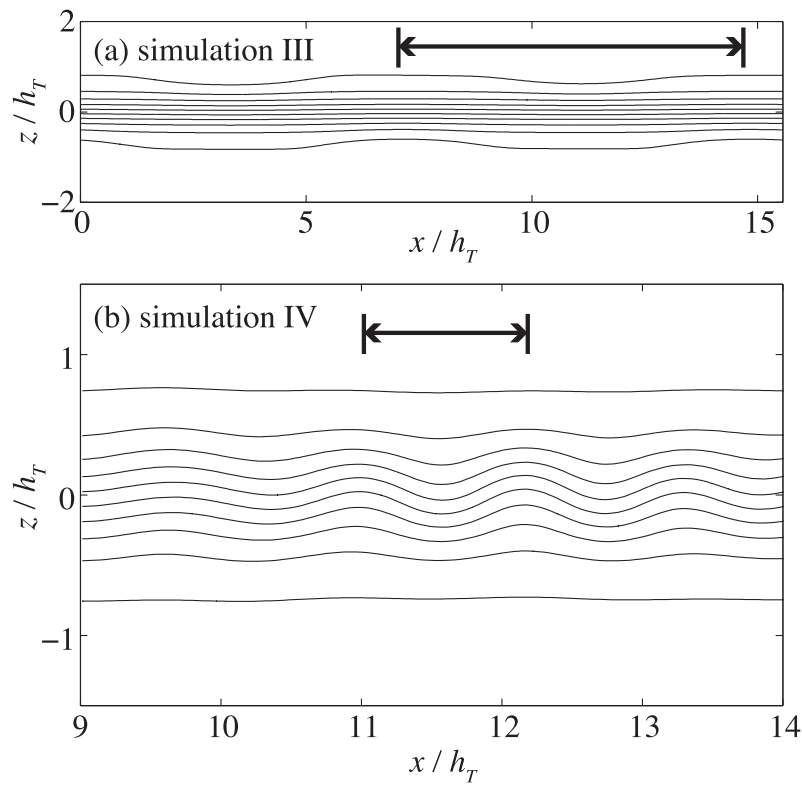

FIG. 9. The $T$ field at the final times of simulations (a) III and (b) IV. The contours are equally spaced between $-0.45 \Delta T$ and $0.45 \Delta T$. The wavelengths $\left(2 \pi h_{T} / \alpha_{\max }\right)$ predicted by linear theory are shown by the length of the horizontal scales. In the case of (b), only part of the domain is plotted.

The growth and eventual breakdown of the instability on the nonstationary interface may be seen by looking at a plot of the time evolution of $h_{T}(t)$. This is shown in Fig. 11a, where we have normalized by the initial thickness $h_{0}=h_{T}(0)$. As the instability develops, a range of different $h_{T}$ are present in the domain at a given $t$ [note that $h_{T}$ is measured using Eq. (14) at each $x$ and $y$ in the domain]. We therefore plot the mean, as well as the 10th and 90th percentiles of $h_{T}$, given by the gray shading. At early times, these curves all fall on one another as $h_{T}$ grows by molecular diffusion, in accordance with (17). The interface enters the unstable region predicted by the linear theory soon after the simulation begins, indicated by the vertical gray strip denoting the time spent inside the DC unstable region. The interface does not experience a growth in the kinetic energy during this time, which can only be seen well after the interface enters the convective unstable region of the diagram (right of the vertical gray strip in Fig. 11b). In other words, the kinetic energy only starts to grow in the convective-type region. The increase in kinetic energy indicates an increasing growth rate over time and eventually saturates after the boundary layers have broken away. The time of the interface breakdown $t_{b}$ is defined as the time of the greatest mean $h_{T}$, which is indicated by the vertical dashed line at $t \approx 2$.

The delay in the growth of the instability when the interfaces are also growing in time may be understood by considering the relative growth rates of these two

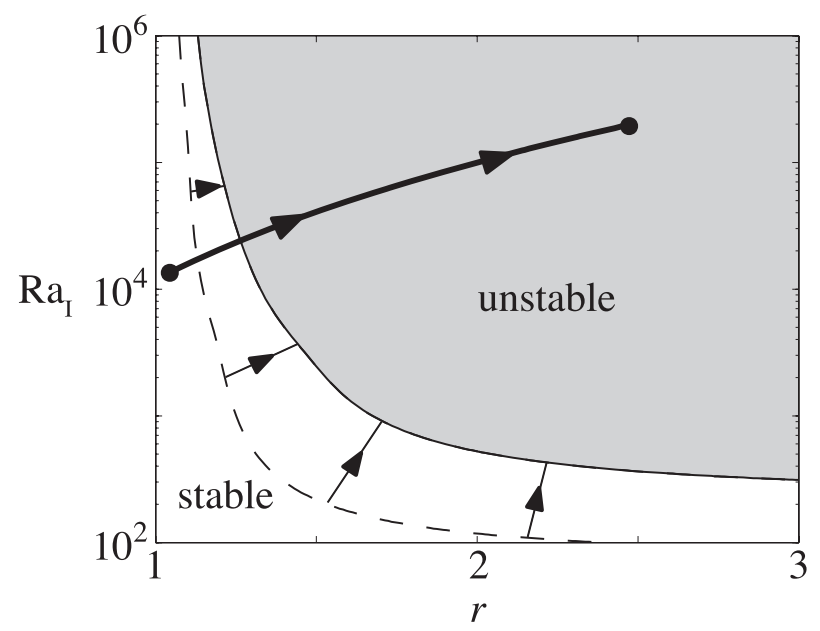

FIG. 10. Trajectory of the interface for simulation $\mathrm{V}$ in the $r-\mathrm{Ra}_{I}$ plane (thick dark line with arrows) up to the time of interface breakdown at $t=t_{b}$ (to be defined more precisely in the text below). The changing position of the stability boundary is given by the dashed line initially and the thin solid line at $t=t_{b}$.

competing processes. The growth rate of the $T$ interface can be defined in dimensional units as

$$
\frac{1}{h_{T}} \frac{d h_{T}}{d t}=\frac{2 \pi \kappa_{T}}{h_{T}^{2}}
$$

where the equality follows from the molecular diffusion of an erf interface expressed in (17). In order for the linear instability to dominate over the growth of the interface, we require $\sigma_{r}$ from linear theory to exceed this interface growth rate. This can be expressed in dimensionless units as the simple relation

$$
\sigma_{r}>2 \pi
$$

Therefore, any instability growing on an interface that is diffusing in time likely requires a (dimensionless) $\sigma_{r}$ in excess of $2 \pi$ to emerge. Because the $\sigma_{r}$ of the DC-type modes are generally less than $2 \pi$, we should instead expect convective-type instabilities in the boundary layers, as is observed. The time at which $\sigma_{r}=2 \pi$ is shown by the solid vertical line in Figs. 11a-c.

The instability can be seen in the $T$ field at the time of breakdown $\left(t=t_{b}\right)$, where a series of plumes have formed in the boundary layers and are in the process of detaching from the interface to be mixed in the upper and lower layers (Fig. 12). Note that the central core of the interface is left undisturbed, because the instability is centered in the boundary layers, as expected.

The results of the nonstationary simulation show that the time-dependent growth of the interface is significant in determining the development of the instability. In the 

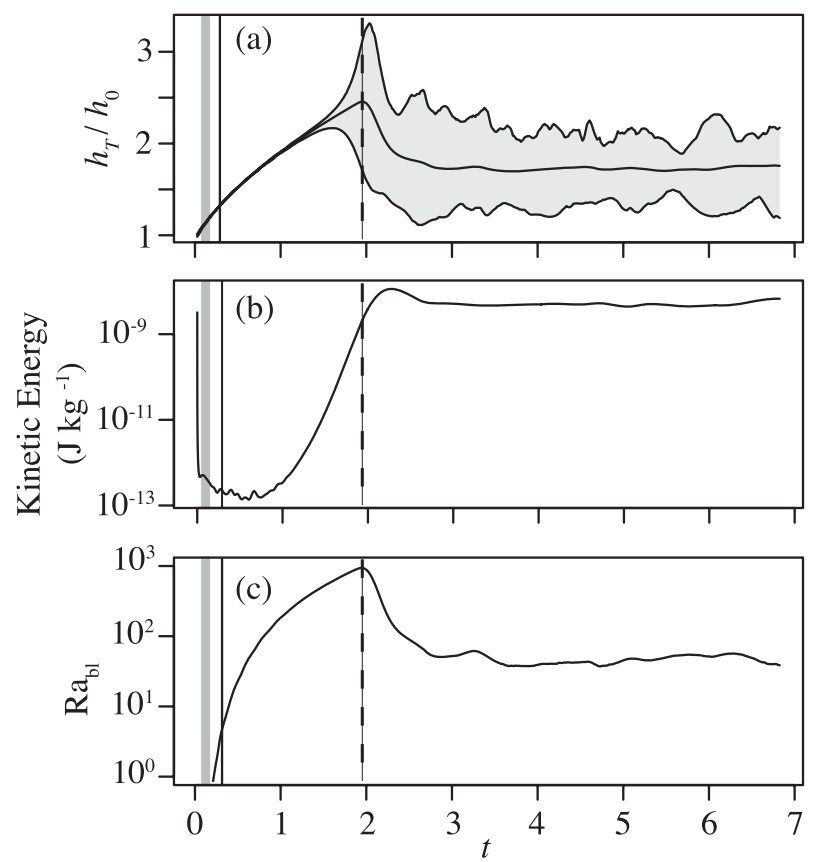

FIG. 11. Time evolution of parameters in the nonstationary simulation $(\mathrm{V})$ as a function of time. The vertical gray strip indicates the time interval of DC instability with stability at earlier times and convective instability at later times. The time at which $\sigma_{r}=2 \pi$ (i.e., when the instability growth rate equals the growth rate of the $T$ interface) is indicated by the vertical solid line. (a) The $T$ interface thickness $h_{T}$, normalized by its initial value $h_{0}$, with the upper and lower lines with gray shading indicating the 90th and 10 th percentiles found within the domain and the central line indicating the mean. (b) Volume-averaged kinetic energy. (c) $\mathrm{Ra}_{\mathrm{bl}}$. In (a)-(c), the time axis has been nondimensionalized by $10^{3} \kappa_{T} / L_{z}^{2}$, and the vertical dashed line indicates the time of peak $h_{T}$, which is denoted $t=t_{b} \approx 2$.

case of simulation $\mathrm{V}$, the interface moves rapidly through the DC-type unstable region composing the stability boundary, which does not contribute to the eventual mode of interface breakdown. We can understand this behavior by requiring that the instability growth rate exceed that of the interface. This predicts that the breakdown is of the convective type and is concentrated in the boundary layers, in agreement with the interpretation of previous investigators (Linden and Shirtcliffe 1978; Newell 1984; Worster 2004). We next investigate whether the transition to instability in the boundary layers can be described by an appropriately defined boundary layer Rayleigh number.

\section{Critical boundary layer Rayleigh number}

As discussed in the introduction, numerous investigators assumed that the nonlinear breakdown of the diffusive interface is governed by a convective-type instability of the unstable boundary layers. In analogy with singlecomponent convection, the criterion for breakdown was taken to be determined by a critical Rayleigh number

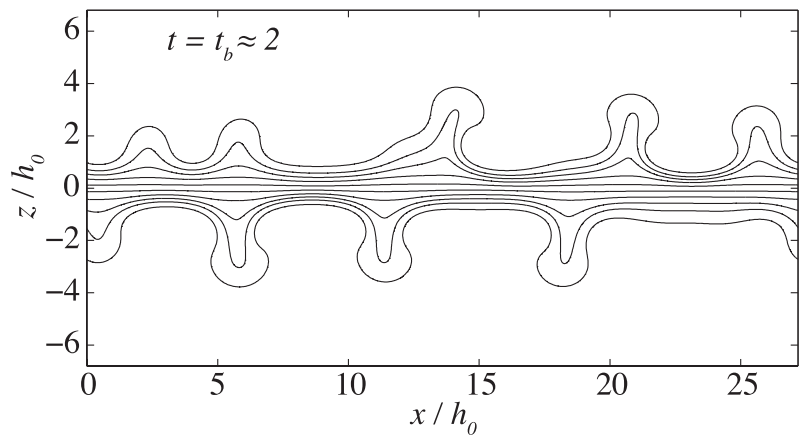

FIG. 12. The $T$ field for the entire domain in simulation $\mathrm{V}$ at $t=t_{b}$, with contour intervals as in Fig. 9.

that is appropriate for the boundary layers, $\mathrm{Ra}_{\mathrm{bl}}=O\left(10^{3}\right)$ (Linden and Shirtcliffe 1978). Furthermore, comparisons with field measurements based on this model have also been attempted (Padman and Dillon 1989; Sánchez and Roget 2007). In this section, we test the use of $\mathrm{Ra}_{\mathrm{bl}}$ in describing the linear stability properties, as well as the nonlinear state of the diffusive interface once convection has begun.

Following Linden and Shirtcliffe (1978), the boundary layer Rayleigh number is defined as

$$
\mathrm{Ra}_{\mathrm{bl}} \equiv \frac{g \delta \rho b^{3}}{\rho_{0} \nu \kappa_{T}}
$$

where $b$ is a representative length scale of the boundary layer (see Fig. 1b), given by

$$
b \equiv\left(h_{T}-h_{S}\right) / 2=h_{T}\left(1-r^{-1}\right) / 2,
$$

and $\delta \rho$ is a representative boundary layer density scale defined by

$$
\delta \rho b \equiv \int_{z_{\mathrm{bl}}}^{L_{z} / 2}\left[\Delta \rho / 2-\left|\bar{\rho}(z)-\rho_{0}\right|\right] d z,
$$

where $\Delta \rho=\Delta S-\Delta T$ and $z_{\mathrm{bl}}$ is determined from $\left|\bar{\rho}\left(z_{\mathrm{bl}}\right)-\rho_{0}\right|=\Delta \rho / 2$ and ensures that the integration in (22) is over the total gravitationally unstable density anomaly in the upper boundary layer. In other words, $z_{\mathrm{bl}}$ is chosen such that all the fluid in the upper boundary layer that is lighter than the mixed layer above, for example, is included in the integration. We can now substitute the dimensional erf profiles, say, of $\bar{T}$ and $\bar{S}$, into (22) to get

$$
\delta \rho b=\frac{h_{T} \Delta T}{2} \int_{\zeta_{\mathrm{b} 1}}^{H / 2}\left[\operatorname{erf}(\sqrt{\pi} \zeta)-R_{\rho} \operatorname{erf}(\sqrt{\pi} r \zeta)+R_{\rho}-1\right] d \zeta
$$




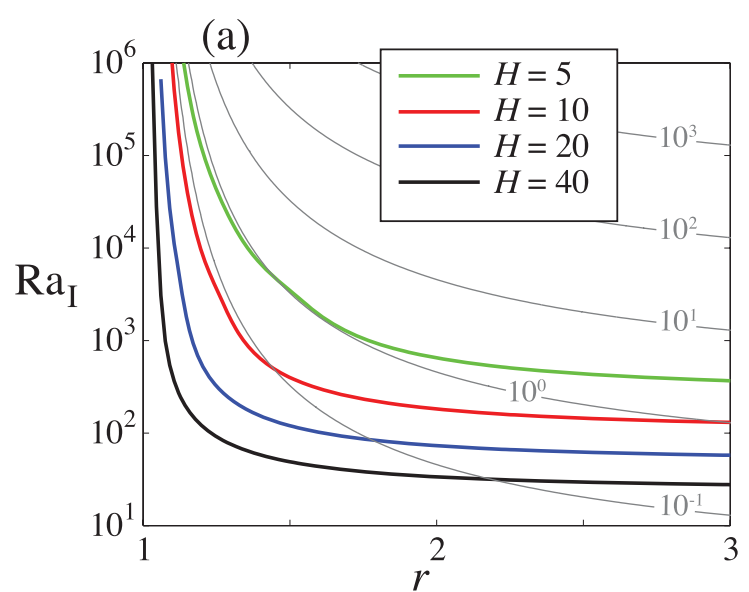

(b)

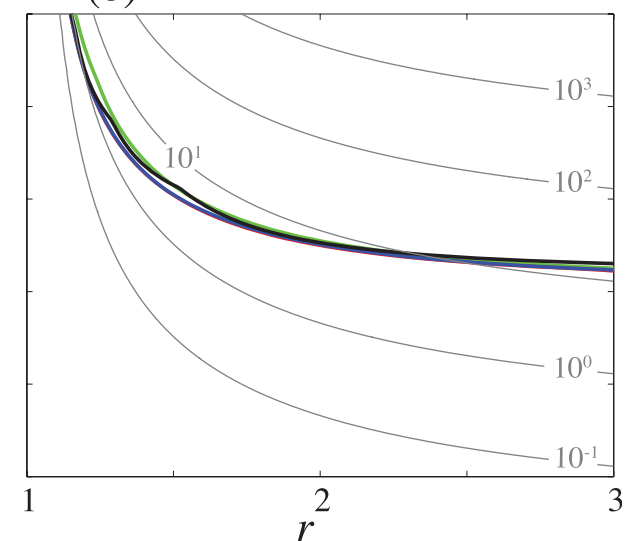

FIG. 13. The boundary between stable and unstable modes as a function of $r$ and $\mathrm{Ra}_{I}$ for $R_{\rho}=3$. (a) The colored curves are plotted for different values of $H$, which can be seen to influence the conditions required for instability. (b) The boundary $\sigma_{r}=2 \pi$ denoting the dominance of the linear instability growth over the growth of the interface by molecular diffusion. Each value of $H$ from (a) is also plotted in (b); however, the curves are indistinguishable from each other. In each of (a),(b), the gray contours give the value of the boundary layer Rayleigh number $\mathrm{Ra}_{\mathrm{bl}}$.

where $\zeta_{\mathrm{bl}}$ can be found from solution of the equation

$\operatorname{erf}\left(\sqrt{\pi} \zeta_{\mathrm{bl}}\right)-R_{\rho} \operatorname{erf}\left(\sqrt{\pi} r \zeta_{\mathrm{bl}}\right)+R_{\rho}-1=0$

It is now possible to express $\mathrm{Ra}_{\mathrm{bl}}$ in terms of the largerscale parameters $\mathrm{Ra}_{I}, R_{\rho}$, and $r$ used in the stability analysis. The link between them can be written in the form

$$
\mathrm{Ra}_{\mathrm{bl}}=\mathrm{Ra}_{I} F\left(R_{\rho}, r\right),
$$

where

$$
F\left(R_{\rho}, r\right) \equiv \frac{1}{8}\left(1-r^{-1}\right)^{2} \cdot \int_{\zeta_{\mathrm{bl}}}^{H / 2}\left[\operatorname{erf}(\sqrt{\pi} \zeta)-R_{\rho} \operatorname{erf}(\sqrt{\pi} r \zeta)+R_{\rho}-1\right] d \zeta
$$

and recall that $\mathrm{Ra}_{I}$ is the interface Rayleigh number (16). It is important to note that a dependence of (25) on $H$ has been neglected. This is justified only if $H \geq 4$ because below this value the boundary layers have reached the domain boundaries and influence the integration.

We are now in a position to ask the question, how applicable is $\mathrm{Ra}_{\mathrm{bl}}$ for describing the onset of instability at a diffusive interface? This question is addressed in Fig. 13, where we have plotted the location of the stability boundary in the $r-\mathrm{Ra}_{I}$ plane from the linear stability analysis for different values of the domain height $H$, at a representative value of $R_{\rho}=3$. Two cases are examined: (i) where the transition to the DC-type unstable modes occurs at $\sigma_{r}=0$ (Fig. 13a) and (ii) where the linear instability growth rate exceeds the growth rate due to diffusion of the interface at $\sigma_{r}=2 \pi$ (Fig. 13b). In the case of (i), Fig. 13a shows that, by varying $H$, there are substantial shifts in the stability boundary, even at values of $H$ as large as 40. It is interesting that this is not so for the convective-type modes that compose the boundary in Fig. 13b, whose curves all collapse.

In each case, it is possible to compare these stability boundaries with contours of $\mathrm{Ra}_{\mathrm{bl}}$ to assess the appropriate critical $\mathrm{Ra}_{\mathrm{bl}}$. (We will restrict ourselves to $H \geq 5$ in order to avoid the dependence of $F$ on $H$.) It can be seen in both cases that the $\mathrm{Ra}_{\mathrm{bl}}$ values that represent the stability boundaries most closely are generally more than two orders of magnitude smaller than the value of $O\left(10^{3}\right)$ previously suggested for the nonlinear breakdown. Furthermore, a single $\mathrm{Ra}_{\mathrm{bl}}$ value is not able to adequately capture the behavior of the stability boundary for the range of $r$ shown and may change by an order of magnitude over $1<r<3$, as can be seen in Figs. 13a,b. We can therefore conclude that a critical $\mathrm{Ra}_{\mathrm{bl}} \approx 10^{3}$ does not accurately describe the linear stability boundary in the boundary layers of a diffusive interface.

That the $\mathrm{Ra}_{\mathrm{bl}}$ required for instability is much less than $10^{3}$ can also be seen in the time-dependent simulation 
(V) shown in Fig. 11c. The instability growth, seen in the kinetic energy plot of Fig. 11 b, is initiated at a $\mathrm{Ra}_{\mathrm{bl}} \ll$ $10^{3}$. It is interesting, however, that the $\mathrm{Ra}_{\mathrm{bl}}$ at the point of the initial breakdown $(t \approx 2)$ is on the order of $10^{3}$, which is what has previously been assumed. At later times $(t>3)$ during active convection, the interface appears to reach a quasi-steady state where $\mathrm{Ra}_{\mathrm{bl}} \approx 50$. The linear stability threshold for time-dependent background profiles, given by $\sigma_{r}=2 \pi$, is $\mathrm{Ra}_{\mathrm{bl}} \approx 5$.

\section{Conclusions}

In this study, we have utilized linear stability analysis and direct numerical simulations (DNS) to examine the conditions under which a diffusive interface will become unstable to small perturbations. It may be thought of as an extension of the classic work on linear profiles of $T$ and $S$ to include the geophysically relevant case of a diffusive interface. Similarity between the linear profile results and the diffusive interface is found when $T$ and $S$ have the same interface thicknesses $(r=1)$. In this case, the instability is centered within the interface and has similar stability properties to the linear profiles.

It has been found, however, that the $T$ interface has a greater thickness than $S(r>1)$, because of the higher $T$ molecular diffusivity (Turner 1973; Marmorino and Caldwell 1976; Linden and Shirtcliffe 1978; Fernando 1989), and this has recently been found in the observations of T. Sommer et al. (2012, unpublished manuscript), as shown in Fig. 2. When this is the case, we have shown that the instability will take place within the diffusive boundary layers on either side of the gravitationally stable interface core, and instability is then possible at the large $R_{\rho}$ values typically observed in the staircases of oceans and lakes (i.e., $2<R_{\rho}<6$, with $R_{\rho}=1.5$ typically the lowest observed; Padman and Dillon 1987; Timmermans et al. 2008; Schmid et al. 2010).

The stability analysis revealed that, within the gravitationally unstable boundary layers, it is possible for instability to take place as either an oscillating DC-type unstable mode or a convective-type unstable mode. The marginally unstable modes that compose the stability boundary are always of DC type. However, the DNS show that the time-dependent growth of the interface is significant in altering the position of the stability boundary. If these time-dependent effects are accounted for by requiring that the instability growth rate exceed that of the interface, then we find that the instability of the boundary layers is of convective type. This finding adds theoretical support to this idea, which has often been assumed.

The boundary layer Rayleigh number $\mathrm{Ra}_{\mathrm{bl}}$ is not found to be a useful parameterization of the stability criterion but may be a good description of the time-dependent conditions under which the breakdown of the interface takes place. Further analysis is required to assess this possibility, and a series of fully three-dimensional DNS have been conducted to examine this issue (Carpenter et al. 2012, manuscript submitted to J. Fluid Mech.). The time dependence of the instability shows that it is not a simple matter to directly apply the stability conditions found here to geophysical observations. However, the findings provide insight into the instability mechanisms, as well as lead to bounds on the parameters governing interface stability.

Acknowledgments. We thank Marco Toffolon, Martin Schmid, and Mary-Louise Timmermans for comments on this manuscript, as well as Bill Smyth and Kraig Winters for providing the DNS code for the simulations. This project has been supported by the Swiss National Science Foundation Grant 200021-122183 (Lake Kivu-Turbulence and double diffusion in permanent stratification). The Swiss National Science Foundation is also acknowledged for partly financing the IPAZIA computational cluster (Project 206021-128754). Part of the salary of the first author was made possible by the EU Project Grant 263287 (FreshMon-High Resolution Freshwater Monitoring: FreshMon GMES Downstream Services).

\section{REFERENCES}

Baines, P., and A. Gill, 1969: On thermohaline convection with linear gradients. J. Fluid Mech., 34, 289-306.

Boehrer, B., S. Dietz, C. von Rohden, U. Kiwel, K. Johnk, S. Naujoks, J. Ilmberger, and D. Lessmann, 2009: Double-diffusive deep water circulation in an iron-meromictic lake. Geochem. Geophys. Geosyst., 10, Q06006, doi:10.1029/2009GC002389.

Fernando, H., 1989: Buoyancy transfer across a diffusive interface. J. Fluid Mech., 209, 1-34.

Hoare, R., 1966: Problems of heat transfer in Lake Vanda, a density stratified Antarctic lake. Nature, 210, 787-789.

Kelley, D., 1990: Fluxes through diffusive staircases: A new formulation. J. Geophys. Res., 95, 3365-3371.

- H. Fernando, A. Gargett, J. Tanny, and E. Özsoy, 2003: The diffusive regime of double-diffusive convection. Prog. Oceanogr., 56, 461-481.

Kundu, P., I. Cohen, and H. Hu, 2004: Fluid Mechanics. 3rd ed. Elsevier, 759 pp.

Linden, P., 2000: Convection in the environment. Perspectives in Fluid Dynamics, G. Batchelor, H. Moffatt, and M. Worster, Eds., Cambridge University Press, 289-345.

— diffusive convection. J. Fluid Mech., 87, 417-432.

Marmorino, G., and D. Caldwell, 1976: Heat and salt transport through a diffusive thermohaline interface. Deep-Sea Res., 23, 59-67.

Neal, V., S. Neshyba, and W. Denner, 1969: Thermal stratification in the Arctic Ocean. Science, 166, 373-374.

Newell, T., 1984: Characteristics of a double-diffusive interface at high density stability ratios. J. Fluid Mech., 149, 385401. 
Newman, F., 1976: Temperature steps in Lake Kivu: A bottom heated saline lake. J. Phys. Oceanogr., 6, 157-163.

Nield, D., 1967: The thermohaline Rayleigh-Jeffreys problem. J. Fluid Mech., 29, 545-558.

Noguchi, T., and H. Niino, 2010: Multi-layered diffusive convection. Part 1. Spontaneous layer formation. J. Fluid Mech., 651, 443-464.

Padman, L., and T. Dillon, 1987: Vertical heat fluxes through the Beaufort Sea thermohaline staircase. J. Geophys. Res., 92, $10799-10806$.

— the Canada Basin diffusive staircase. Deep-Sea Res., 36, 531-542.

Sánchez, X., and E. Roget, 2007: Microstructure measurements and heat flux calculations of a triple-diffusive process in a lake within the diffusive layer convection regime. J. Geophys. Res., 112, C02012, doi:10.1029/2006JC003750.

Schmid, M., A. Lorke, C. Dinkel, G. Tanyileke, and A. Wüest, 2004: Double-diffusive convection in Lake Nyos, Cameroon. Deep-Sea Res., 51, 1097-1111.

—, M. Busbridge, and A. Wüest, 2010: Double-diffusive convection in Lake Kivu. Limnol. Oceanogr., 55, 225-238.

Shirtcliffe, T., 1973: Transport and profile measurements of the diffusive interface in double diffusive convection with similar diffusivities. J. Fluid Mech., 57, 27-43.
Smyth, W., and S. Kimura, 2007: Instability and diapycnal momentum transport in a double-diffusive, stratified shear layer. J. Phys. Oceanogr., 37, 1551-1565.

— J. Nash, and J. Moum, 2005: Differential diffusion in breaking Kelvin-Helmholtz billows. J. Phys. Oceanogr., 35, 1004 1022.

Stern, M., 1960: The salt-fountain and thermohaline convection. Tellus, 2, 172-175.

Timmermans, M.-L., J. Toole, R. Krishfield, and P. Winsor, 2008: Ice-tethered profiler observations of the double-diffusive staircase in the Canada Basin thermocline. J. Geophys. Res. 113, C00A02, doi:10.1029/2008JC004829.

Turner, J., 1973: Buoyancy Effects in Fluids. Cambridge University Press, 367 pp.

Veronis, G., 1965: On finite amplitude instability in thermohaline convection. J. Mar. Res., 23, 1-17.

Winters, K., J. MacKinnon, and B. Mills, 2004: A spectral model for process studies of rotating, density-stratified flows. J. Atmos. Oceanic Technol., 21, 69-94.

Worster, M., 2004: Time-dependent fluxes across double-diffusive interfaces. J. Fluid Mech., 505, 287-307.

You, Y., 2002: A global ocean climatological atlas of the Turner angle: Implications for double-diffusion and water-mass structure. Deep-Sea Res., 49, 2075-2093. 